\title{
Individual and age-related differences in children's working memory
}

\author{
H. LEE SWANSON \\ University of California, Riverside, California
}

\begin{abstract}
The purpose of this study was to investigate the degree to which age-related and individual differences in children's working memory (WM) are due to a general or task-specific capacity system. Experiment 1 correlated children's $(N=146$; age range 5-19 years) verbal and visual-spatial working memory performance with various intelligence and achievement measures. The results supporting a general system were that (1) visual-spatial and verbal WM measures were significantly intercorrelated with and without age partialed out and (2) both verbal and visual-spatial WM measures were significantly correlated with diverse achievement and intelligence measures. Experiment 2 compared three age groups $(N=192 ; 7-, 10$, and 13-year-olds) on working-memory performance tasks under initial, enhanced (cued), and maintenance conditions. The results supporting a general capacity system were that (1) age-related performance differences in WM were found on all conditions and not isolated to specific processes, (2) the maintenance measures (high-load condition) predicted the variance better in age-related performance than process measures, and (3) although individual differences in WM performance reflected two independent operations, these operations produced similar correlations to achievement within age groups. Overall, the results support a general capacity explanation of age-related and individual differences in children's WM performance.
\end{abstract}

The present study was designed to investigate the source of age-related and individual differences in children's working memory (WM). The central question in this study was whether age-related and individual differences in working memory (WM) among children reflect a general or a specialized system. A related question is whether the same WM processes responsible for age-related differences are also responsible for individual differences within age groups. It is of further interest to determine whether age-related or individual differences in WM performance can be modified and whether the remaining sources of variance can be attributed to the processing or storage of information. As in previous studies (e.g., Just \& Carpenter, 1992; Salthouse, Mitchell, Skovronek, \& Babcock, 1989; Turner \& Engle, 1989), working memory is defined in this study as the preservation of information while simultaneously processing the same or other information.

Two issues are involved in identifying the source of age-related and individual differences in children's WM. First, there is no consensus about whether age-related and individual differences in WM reflect a domain-specific or common central executive system. For example, Daneman and Tardiff (1987) have recently suggested that WM is domain specific, not based on a common system. The

This research was partially funded by a SSHRC grant to the author at the University of British Columbia and Peloy Endowment Funds from the University of California. Correspondence should be addressed to H. L. Swanson, School of Education, University of California, Riverside, CA 92521. primary evidence for their conclusion was that measures of verbal WM, not visual-spatial WM, were significant predictors of reading comprehension (see Engle, Cantor, \& Carullo, 1992, for a review). Daneman and Carpenter (1980) have also argued that the overall executive capacity of WM does not differ across individuals. Individuals do differ, however, in the storage component of WM as a consequence of how much attention their reading process requires (Daneman, 1987; Daneman \& Green, 1986). In contrast, Baddeley (1986) has hypothesized that there are at least two distinct store regions concerned with auditory-verbal and visual-spatial information, and a single central executive system that is responsible for processing information. As memory tasks place more demands on concurrent processing, performance becomes dependent on the common central processor to process and manipulate information (see Morris, Craik, \& Gick, 1990; Salthouse \& Babcock, 1991; Turner \& Engle, 1989). Thus, correlations between seemingly diverse WM measures are higher when executive processing is required than when it is not.

Second, it is unclear whether individual and age-related differences in children's WM are primarily related to processing efficiency or capacity. Several studies suggest that age-related differences in children's WM are related to processing efficiency (e.g., Case, 1985; Case, Kurland, \& Goldberg, 1982; see Howe \& Rabinowitz, 1990 , for a review). For example, Case (1985) has characterized young children's WM capacity as taxed by processing demands, so little space is available for storage, and, therefore, WM performance is poor. However, as children gain experience, executive functions, strategies, 
and other processes become more efficient, thus freeing WM for storage (see Bjorklund \& Harnishfeger, 1990, for a review). In contrast, Turner and Engle (1989) have suggested that "working-memory may be a unitary individual characteristic, independent of the nature of the task in which the individual makes use of it" (p. 150). In support of this general-capacity hypothesis, Engle and colleagues (e.g., Cantor, Engle, \& Hamilton, 1991; Turner \& Engle, 1989) found that visual-spatial tasks correlated with reading comprehension scores as highly as did verbal WM tasks. Their findings suggest that WM capacity is not dependent on the particular strategy used to accomplish the task at hand, which further suggests that visual-spatial and verbal WM measures tap the same underlying system.

In contrast to a general-capacity hypothesis, as reflected in Engle's work, a processing-efficiency framework is frequently used to explain age-related and individual differences in children's WM performance. One reason for this is that although some researchers accept the notion of WM capacity, they question the existence of a general pool, instead proposing independent or specialized systems (e.g., Brainerd \& Reyna, 1989, 1990; however, see Dempster, 1993a, and Howe, Rabinowitz, \& Grant, 1993, for discussion). It is further argued that support for a general-capacity model requires evidence that a global mechanism accounts for most of the variance in age-related change (Salthouse, 1990). Perhaps the most damaging criticism for a general WM system has been the weak correlations between memory measures and higher order tasks, such as problem solving (Brainerd \& Reyna, 1989). However, such independence may be related to statistical power (Chapman \& Lindenberger, 1992); that is, it may be a consequence of independent sampling of two tasks on separate occasions (see Howe et al., 1993, p. 741, for a review), and memory load (Howe \& Rabinowitz, 1991). Thus, although the link between working memory and performance on other cognitive measures is under debate (see also Bjorklund \& Harnishfeger, 1990; Howe et al., 1993), more specific hypotheses related to a capacity model are necessary if it is to remain as a possible explanation of age-related and individual differences in children's WM performance.

Borrowing from several authors (Bjorklund \& Harnishfeger, 1990; Howe et al., 1993, Salthouse, 1990; Salthouse et al., 1989), two specific hypotheses are considered in the present study. First, WM tasks are relevant to many different cognitive tasks. This hypothesis is important for distinguishing a general system from a process or specific system because WM resources are "postulated to have greater across-task relevance than the processes or components in information-processing models of specific tasks" (Salthouse, 1990, p. 120). Second, WM accounts for a significant proportion of variance in agerelated performance. It seems unlikely that a capacity hypothesis would provide an adequate account of agerelated or individual differences in children's cognitive performance unless it were found to be responsible for a substantial proportion of the variance. Salthouse (1990) indicated that WM should account for at least one third to one half of the total age-related variance if a capacity model is to be taken seriously. However, both a general process and capacity model would postulate relevance across multiple measures. To unravel these two interpretations, two additional hypotheses are tested here. One hypothesis, a general-processing efficiency model, predicts that measures of processing efficiency will account for the majority of variance in age-related performance (see, e.g., Case, 1985). The other hypothesis, a generalcapacity model, predicts that age-related performance is best predicted by high-memory demand conditions (e.g., Salthouse \& Babcock, 1991).

\section{EXPERIMENT 1}

Experiment 1 investigated whether diverse WM measures share a general system, as well as whether WM underlies age-related and individual performance on a number of diverse cognitive tasks. It was hypothesized that WM is generalized across tasks in children's performance, and therefore reasonable correlations should be found between WM measures and a multitude of cognitive measures. If the correlational patterns between diverse WM tasks and cognitive measures are generalized across age and individuals, then one may assume that a general WM system is in operation. In contrast, a specificprocess model would be supported if verbal WM tasks yield significant correlations that are isolated to reading and visual-spatial WM tasks yield significant correlations to mathematics. Experiment 1 also serves as a validation of WM tasks because previous studies on agerelated performance have reported poor reliability and intercorrelations among WM measures (see Salthouse, 1990 , for a review). Thus, it is assumed that to emphasize variance associated with the WM construct, and to minimize variance associated with a particular procedure or material to measure WM, it is desirable to obtain multiple measures of WM performance (see Salthouse \& Babcock, 1991, for review of conflicting results with isolated measures).

\section{Method}

Subjects. Subjects were 146 children ( 75 females, 71 males) selected from various school districts surrounding a large northwest city. Mean age was 10.32 years $(S D=3.15$; range $=5$ to 19 years $)$. These subjects were drawn from a project (Swanson, 1995) to standardize WM measures.

Aptitude, achievement, and short-term memory (STM) measures. One achievement test and three standardized aptitude measures were administered: the Peabody Individual Achievement Test (PIAT; Dunn \& Markwardt, 1989), the Kaufman Assessment Battery for Children (K-ABC; Kaufman \& Kaufman, 1983), the Detroit Tests of Learning Aptitude-Second Edition (DTLA-2; Hammill, 1985), and the Peabody Picture Vocabulary Test (PPVT; Dunn, 1981). Mean scores for the subjects on the various tests administered are provided in Table 1. However, because of time limitations, not all subjects were administered all measures. Thus, sample size for each measure is shown in Table 1.

Five subtests from the PIAT were administered: Mathematics, Reading Recognition, Reading Comprehension, Spelling, and Gen- 
Table 1

Mean Scores on Aptitude, Achievement, and Working-Memory Measures

\begin{tabular}{|c|c|c|}
\hline \multicolumn{3}{|l|}{ Measure } \\
\hline \multicolumn{3}{|l|}{$\overline{1 . \mathrm{K}-\mathrm{ABC}(N=145)}$} \\
\hline Simultaneous Processing & $\begin{array}{c}41.41 \\
(99.18)\end{array}$ & $\begin{array}{r}12.06^{*} \\
(14.66)\end{array}$ \\
\hline Successive Processing & $\begin{array}{c}75.37 \\
(99.59)\end{array}$ & $\begin{array}{c}20.00^{*} \\
(14.43)\end{array}$ \\
\hline 2. $\operatorname{PPVT}(N=99)$ & 102.41 & $12.52 \dagger$ \\
\hline \multicolumn{3}{|l|}{ 3. DTLA-2 $(N=127)$} \\
\hline Sentence Imitation & $\begin{array}{c}13.25 \\
(100.69)\end{array}$ & $\begin{array}{r}4.83^{*} \\
(14.54)\end{array}$ \\
\hline Object Sequence & $\begin{array}{c}25.71 \\
(99.42)\end{array}$ & $\begin{array}{r}4.63^{*} \\
(14.04)\end{array}$ \\
\hline \multicolumn{3}{|l|}{ 4. $\operatorname{PIAT}(N=104)$} \\
\hline Mathematics & 100.61 & $12.13 \dagger$ \\
\hline Reading Recognition & 101.11 & $11.98 \dagger$ \\
\hline Reading Comprehension & 101.53 & $12.46 \dagger$ \\
\hline Spelling & 100.78 & $13.67 \dagger$ \\
\hline General Information & 101.05 & $14.51 \dagger$ \\
\hline 5. Sentence Span Task $(N=54)$ & 1.20 & $1.12 \ddagger$ \\
\hline \multicolumn{3}{|l|}{ 6. Working-Memory Battery } \\
\hline Rhyming $(N=138)$ & 1.86 & $1.23 \ddagger$ \\
\hline Visual Matrix $(N=144)$ & 3.40 & $1.54 \ddagger$ \\
\hline Auditory Digit Sequence $(N=145)$ & 2.05 & $1.32 \ddagger$ \\
\hline Mapping and Directions $(N=145)$ & 1.68 & $1.35+$ \\
\hline Story Recall $(N=145)$ & 4.51 & $3.75 \ddagger$ \\
\hline Picture Sequencing $(N=133)$ & 1.98 & $1.31 \ddagger$ \\
\hline Phrase Recall $(N=139)$ & .87 & $.84 \ddagger$ \\
\hline Spatial Organization $(N=34)$ & 1.74 & $1.84 \ddagger$ \\
\hline Semantic Association $(N=145)$ & 1.35 & $1.09 \ddagger$ \\
\hline Semantic Categorization $(N=134)$ & 1.85 & $1.16 \ddagger$ \\
\hline Nonverbal Sequencing $(N=140)$ & 4.51 & $5.62 \ddagger$ \\
\hline
\end{tabular}

Note-Standard scores (in parentheses) are approximations because some children are older than those in the standardization sample. K-ABC, Kaufman Assessment Battery for Children; PPVT, Peabody Picture Vocabulary Test; DTLA-2, Detroit Tests of Learning Aptitude Second Edition; PIAT, Peabody Individual Achievement Test. *Raw score. †Standard score. ‡Span score.

eral Information. A multiple-choice format is used in all subtests except General Information, where short-answer response is required. Reliabilities on the subtests vary from .52 to .92 . The K-ABC provides an intellectual mental processing scale that measures sequential and simultaneous processing. Because some subjects were older than the norms for the test (chronological age $>12$ ), only raw scores were computed for the correlational analysis. The PPVT requires the examiner to read a stimulus word, and the child responds by pointing to the picture illustrating the word. Items are arranged in order of ascending difficulty. Reported reliabilities vary from .83 to .95 .

Two standardized subtests from the DTLA-2 were selected to assess short-term memory. The tasks were selected because of their high reliability, uncharacteristic of most short-term memory measures (see Dempster, 1985, for a review). For the Sentence Imitation subtest, words are read aloud to the subjects, one word every second. After hearing the sentence, the subject repeats the sequence Sentences increase in word length from 6 to 19 words. The Object Sequence subtest presents a series of pictures that increase in set size. After the pictures are withdrawn, the child is asked which of the previously presented pictures is out of order and to reorder them correctly. Reliabilities on the subtests vary from .84 to .97 .

Working-memory battery. The battery of WM tasks is described elsewhere (Swanson, 1992, 1993, 1995). All WM tasks were designed to conform with Baddeley's (1986) definition that they "require simultaneous processing and storage of information" and "measure various contents" (pp. 34-35). A critical feature of the WM tasks in Experiment 1 is that they require the maintenance of some information during the processing of other information. Consistent with Daneman and Carpenter's (1980) working-memory measures, the processing of information is assessed by asking children simple questions about the to-be-remembered material (storage + processing demands), whereas storage is assessed by accuracy of item retrieval (storage demands only). The process question generally requires a simple recognition of new and old information, and is analogous to Daneman and Carpenter's task, which requires a yes/no response to previously presented information. It is important to note that the difficulty of the processing question remains constant within task conditions, thereby allowing the source of performance differences to reflect increases in storage demands. These tasks reflect a broad array of processing (verbal and visualspatial) demands, resource demands (semantic and episodic), and retrieval conditions (prospective and retrospective). The rationale and classification of each task as a function of verbal or visualspatial processing are provided below (the task number refers to the order in which tasks were administered).

\section{Verbal Processing}

Task 1-rhyming. The purpose of this task was to assess the child's recall of acoustically similar words. The child listens to sets of words that rhyme. Each successive word in the set is presented every $2 \mathrm{sec}$. Nine word sets range from 2 to 14 monosyllabic words. Before the subject recalls the words, he/she is asked whether a particular word was included in the set. For example, the subject is presented the words lip-slip-clip and then asked if ship or lip was presented in the word set. The subject is then asked to recall the previously presented words (lip-slip-clip) in order. The dependent measure is the number of sets recalled correctly (range $=0-9$ ).

Task 3-auditory digit sequence. The purpose of this task was to assess the child's ability to remember numerical information embedded in a short sentence. Prior to stimulus presentation, the child is shown a figure depicting four strategies for recalling numerical information. These strategies are pictorial representations of rehearsal, chunking, associating, and elaborating of information. (A verbal description of strategies, prior to administration of targeted items, is the same format as that used for Tasks $4,7,8,10$, and 11.) After all strategies have been explained, children are presented numbers in a sentence context. A sample sentence (Item 3) is, "Now suppose somebody wanted to have you take them to the supermarket at 8651 Elm Street..." Numbers are presented at the rate of one every $2 \mathrm{sec}$. Children are then presented a process question, "What is the name of the street?" They are then told that they will have to recall the numbers in the sentence in order shortly after they select from (point to) a pictorial array (as shown in Figure 1, Swanson, 1993) the strategy that best approximates how they will attempt to remember the information. No further information about the strategies shown in the picture is provided. The range of recall difficulty is 3 digits to 14 digits, and the dependent measure is the number of sets correctly recalled ( 0 to 9 ).

Task 5 -story recall. The purpose of this task was to assess the child's ability to remember a series of episodes presented in a paragraph. The experimenter reads a paragraph, asks a process question, and then asks the child to recall all the story events in order. The paragraph is a 12-sentence story in which each sentence includes two idea units and 8 to 11 words. The paragraph is related to the famous battle of the Armada, in which a small fleet of English ships beat the Spanish fleet. For the process question, the child is asked, "Who won the battle, the large or small ships?" The dependent measure is number of sentences recalled correctly and in order (range of 0 to 11). For a sentence to be recalled correctly, it must include two idea units and occur in the correct order.

Task 7-phrase sequence. The purpose of this task was to determine the child's ability to remember isolated phrases. The child is instructed to remember all phrases, but not necessarily in order. 
An increasing number of phrases is presented. After each presentation, a process question is asked, and the child is informed that he/she will have to remember this information shortly after selecting the best strategy to help him/her remember the material. The strategies are pictorial representations of elaborating, indexing, associating, and chaining information. A sample sequence of phrases (Set 3 ) is a flowing river, a slow bear, a growing boy, a gripping tire. A sample process question is, "Are the words about a bear or boat?" The range of difficulty is 2 to 12 phrases. The dependent measure is the number of sets recalled correctly (range of 0 to 9 ).

Task 9-semantic association. The purpose of this task was to determine the child's ability to organize words into abstract categories. The child is presented some words (one every $2 \mathrm{sec}$ ), asked a process question, and asked to recall the words that go together. For example, some words to be categorized (for Set 3) include shirt, saw, pants, hammer, shoes, and nails. The child is directed to retrieve the words that go together (i.e., shirt, pants, and shoes; saw, hammer, and nails). The process question is, "Which word, saw or level, was said in the list of words?" Thus, the task requires the child to transform information that has been encoded serially into categories during the retrieval phase. The child is told that the words can be recalled in any temporal order within a particular category, provided the words are related to the appropriate category. The range of difficulty is two categories of two words to five categories of four words. The dependent measure is the number of sets recalled correctly (range of 0 to 8 ).

Task 10-semantic categorization. The purpose of this task was to determine the child's ability to remember words within categories. One word is presented every $2 \mathrm{sec}$ and the child is told that she/he will have to remember this information shortly after telling the examiner how she/he will attempt to remember the material The child is asked to recall the category name first, and then any word that went with that category. Prior to recall of the words, however, the child is asked a process question and then asked to select a strategy that will facilitate recall of the words. A sample item (Item 3 ) is job, teacher, fireman, policeman, season, summer, winter, fall. A sample process question is, "Which word, soldier or summer, was presented?" The four pictorial examples of strategies include top-down superordinate organization, interitem discrimination, interitem associations, and subjective organization. The range of difficulty for the sets is from two words within a category to eight categories with three words within each category. The score range is 0 to 8 sets.

\section{Visual-Spatial Processing}

Task 2-visual matrix. The purpose of this task was to assess the child's ability to remember visual sequences within a matrix The child is presented a series of dots in a matrix and is allowed $5 \mathrm{sec}$ to study the matrix. The matrix is removed and the child is asked a process question: "Are there any dots in the first column?" To insure the understanding of the word column, the experimenter points to the first column on a blank matrix (a grid with no dots). After the child answers the process question, he/she is asked to draw the dots in the correct boxes on the blank matrix. The task difficulty ranges from a matrix of 4 squares and 2 dots to a matrix of 45 squares and 12 dots. The dependent measure is the number of matrices recalled correctly (range of 0 to 11 )

Task 4-mapping and directions. The purpose of this task was to determine whether the child could remember a sequence of directions on a map that is void of labels. As shown in Figure 2 of Swanson (1993), the experimenter presents the child with a street map with lines connected to a number of dots that illustrate the direction a bike would go to get through the city. The dots represent stoplights and the lines the direction the bicycle should go. The map is removed after $10 \mathrm{sec}$. The child is then asked a process question and to point to the strategy (picture) that he/she thinks he/she will use to remember the street directions. Finally, the child is asked to draw on another map the street directions (lines) and stoplights (dots) on a blank map. The process question is, "Were there any dots in the first street [column]?" With the same pictorial format as that in Task 3, strategies are pictorial representations of elemental, global, sectional, or backward processing of patterns. Task difficulty ranges from 4 to 19 dots. The dependent measure is the number of maps drawn correctly (range of 0 to 9 ).

Task 6-picture sequence. The purpose of this task was to assess the child's ability to remember an increasing sequence of shapes in order. Pictures of shapes (e.g., * ${ }^{*}$ ) are presented in different orders and positions on a series of cards and displayed for $30 \mathrm{sec}$. The cards are gathered, a process question is asked, and then the child is instructed to arrange the cards in the correct sequence. The process question is, "Is this card [distractor card] or this card [card selected from another set] the one I presented?" The dependent measure is the number of sets of cards correctly reproduced. The set size varies from 3 to 15 and scores vary from 0 to 9 .

Task 8-spatial organization. The purpose of this task was to determine the child's ability to remember the spatial organization of cards with pictures of various shapes. These cards are ordered in a top-down fashion (see Figure 3 of Swanson, 1993). The presentation of this task includes five steps: (1) A description of each strategy is provided; (2) the experimenter presents the sequenced cards in their correct order and allows the child $30 \mathrm{sec}$ to study the layout; (3) the experimenter gathers up the cards, shuffles them, then asks a process question; (4) the experimenter asks the child to select a strategy that he/she will use to remember the cards; and (5) the child is directed to reproduce each series of cards in the order in which they were given. For the process question, prior to the child placing the cards in the correct rows and order, the experimenter takes out the first card (row 1) and last card (row 8) and asks, "Which card came first?" As in Task 3, the strategies to be selected are pictorial representations focusing on imagery, pattern similarity, pattern dissimilarity, and visual sequencing. The dependent measure is the number of rows recalled correctly (range of 0 to 8 ).

Task 11-nonverbal sequencing. The purpose of this task was to determine the child's ability to sequence a series of cards with pictures of nonsense shapes (see Swanson, 1993, for discussion of shapes). The child is presented a series of cards in which the proper order is not provided by the experimenter. The child is allowed to organize the cards into any rows that he/she would like with the stipulation that a certain number of cards is included in each row. The first row must have one card, the second row two cards, the third row four cards, the fourth row six cards, and the fifth and sixth row eight cards. The child is given 2 min to place the cards in rows. After the rows have been established, and the child has studied them for $30 \mathrm{sec}$, the cards are gathered up and then he/she is asked a process question. The process question asks, "Is this card [card in the first row] or this card [distractor card randomly chosen] the one you put into the first row?" The child is then asked to select the picture that best represents how she/he is planning to remember this sequence. The four strategies depicted in the illustration include an image of hierarchical association, subordinate association, global sorting, and bottom-up sequencing. The experimenter then inserts two distractor cards, shuffles the cards, and asks the child to reproduce the card arrangement previously studied. The range of difficulty is the recall of one card per row to eight cards per row. The dependent measure is number of cards placed correctly in each row (range of 0 to 6 ).

Reliability analysis. Reliability estimates for children's memory span scores were derived from Cronbach's alpha formula as measures of internal consistency. Reliability estimates on memory span scores for the 11 subtests varied from .75 to .98 , whereas overall reliability (summed score across tasks) was .96 .

Sentence span task. The psychometric characteristics of the WM tasks were validated. Construct validity was established by correlating the measures with the sentence span task and criterionrelated validity was established on the measures of intelligence and 
Table 2

Pearson Product Moment Correlations Between Working-Memory Measures and Short-Term Storage Measures

\begin{tabular}{lccc}
\hline \multicolumn{1}{c}{ Measures } & $\begin{array}{c}\text { Sentence } \\
\text { Span } \\
(N=54)\end{array}$ & $\begin{array}{c}\text { Sentence } \\
\text { Imitation } \\
(N=127)\end{array}$ & $\begin{array}{c}\text { Object } \\
\text { Sequence } \\
(N=127)\end{array}$ \\
\hline 1. Rhyming & $.41 \dagger$ & .08 & .12 \\
2. Visual Matrix & $.43 \dagger$ & $.30 \dagger$ & $.21^{*}$ \\
3. Auditory Digit Sequence & $.41 \dagger$ & $.21 \dagger$ & .10 \\
4. Mapping and Directions & $.72 \dagger$ & $.24^{*}$ & .06 \\
5. Story Recall & $.81 \dagger$ & $.38 \dagger$ & $.26 \dagger$ \\
6. Picture Sequencing & $.53 \dagger$ & .13 & .07 \\
7. Phrase Sequence & $.56 \dagger$ & .07 & .05 \\
8. Spatial Organization & $.53 \dagger$ & .11 & .15 \\
9. Semantic Association & $.57 \dagger$ & $.20 \dagger$ & .07 \\
10. Semantic Categorization & $.62 \dagger$ & .03 & $.21^{*}$ \\
11. Nonverbal Sequencing & $.50 \dagger$ & -.09 & .07 \\
\hline
\end{tabular}

${ }^{*} p<.05 . \quad \dagger p<.01$.

achievement. Discriminant validity was established by comparing the correlation of WM and short-term memory with achievement and processing measures. All WM tasks were compared on the children's adaptation (Swanson, Cochran, \& Ewers, 1989) of Daneman and Carpenter's (1980) sentence span measure. Materials for the adapted sentence span task are unrelated declarative sentences, 7 to 10 words in length. Sentences are read to children and they are to follow along silently. The task requires them to recall the last word of several sentences, as well as to answer a comprehension question about a sentence. The sentences are arranged randomly into sets of two, three, four, or five. Cronbach's coefficient alpha for the measure is .92 .

Procedure. All tests were administered individually. Testing was done in approximately three $1-\mathrm{h}$ sessions at different periods. All psychometric tests were administered first, followed by the memory tasks. Administration of the psychometric measures followed the test manuals. All items on WM tasks were administered until a process question was missed or until an error in retrieval occurred.

\section{Results}

Table 1 presents the mean scores and standard deviations for all measures. All raw scores were converted to $z$-scores based on the total sample for the subsequent analysis. Table 2 shows the Pearson product moment correlations between the WM battery, sentence span task, and the two short-term memory (STM) measures. As shown in Table 2, the magnitude of the correlations was higher $(r>.50)$ between WM measures and the sentence span measure than between the WM measures and the STM measures. All WM tasks were significantly related to the sentence span task, whereas less than half the WM tasks were significantly related to the majority of shortterm memory measures. As shown, both visual-spatial and verbal WM correlated with the sentence span measure. Because the individual tasks on the WM battery were significantly related to the sentence span task, scores from the battery were summed across tasks in the subsequent analysis. The partial correlation coefficient between the total composite score of the WM battery and the sentence span measure, with the influence of age partialed out, was significant $[r(52)=.60, p<.001]$. Correlations between the sentence span measure and the STM measures (Sentence Imitation and Object Sequence), with the influence of age partialed out, were .17 and .07 ( $p \mathrm{~s}>$ .05 ), respectively. In general, the results suggest that the WM measures have high construction and divergent validity.

Intercorrelations. Pearson product moment correlations between the WM task scores are shown in Table 3. The average correlation, without the effects of age removed, was significant $[r(131)=.36, p<.001]$. Table 3 also shows the correlations related to age. On the basis of the number of significant correlations, it would seem that increases in age inflate the intercorrelations between WM measures. However, the average intercorrelation remained significant with age partialed out $[r(130)=.20$, $p<.05]$. In addition, the mean partialed and nonpartialed coefficients did not vary significantly (Fisher $z$ score transformation, $z=1.41, p \mathrm{~s}>.05$ ), suggesting that a general system has an influence at both an individualdifference and age-related level.

Correlations with various cognitive domains. Because WM and performance on cognitive measures improved with age, age was partialed out in the subsequent analysis to better isolate individual differences. As shown in Table 4, the WM composite score from the battery was significantly correlated with all intelligence and achievement measures. Further, this pattern of correlations was comparable to that of the sentence span measure. As shown, the span score from the complete working memory

Table 3

Intercorrelation Between Working-Memory Tasks

\begin{tabular}{|c|c|c|c|c|c|c|c|c|c|c|c|c|}
\hline & 1 & 2 & 3 & 4 & 5 & 6 & 7 & 8 & 9 & 10 & 11 & 12 \\
\hline 1. Rhyming & & .26 & .54 & .32 & .24 & .39 & .35 & .36 & .34 & .32 & .03 & .44 \\
\hline 2. Visual Matrix & & & .42 & .55 & .59 & .41 & .08 & .40 & .36 & .33 & .21 & .56 \\
\hline 3. Auditory Digit Sequence & & & & .39 & .49 & .50 & .39 & .33 & .49 & .53 & .06 & .58 \\
\hline 4. Mapping and Directions & & & & & .59 & .39 & .29 & .36 & .36 & .34 & .29 & .61 \\
\hline 5. Story Recall & & & & & & .51 & .31 & .40 & .33 & .38 & .24 & .74 \\
\hline 6. Picture Sequence & & & & & & & .42 & .43 & .44 & .46 & .15 & .61 \\
\hline 7. Phrase Sequence & & & & & & & & .35 & .32 & .39 & .08 & .38 \\
\hline 8. Spatial Organization & & & & & & & & & .33 & .26 & .20 & .54 \\
\hline 9. Semantic Association & & & & & & & & & & .59 & .02 & .42 \\
\hline 10. Semantic Categorization & & & & & & & & & & & .29 & .53 \\
\hline 11. Nonverbal Sequencing & & & & & & & & & & & & .31 \\
\hline
\end{tabular}

Note - If $r>.16, p<.05$. If $r>.21, p<.01$. If $r>.26, p<.001$. 
Table 4

Pearson Product Moment Correlations Between Working-Memory Measures, Aptitude, and Achievement Measures With Age Partialed Out

\begin{tabular}{lcc}
\hline & $\begin{array}{c}\text { Working-Memory } \\
\text { Composite }\end{array}$ & $\begin{array}{c}\text { Sentence Span } \\
\text { Task }\end{array}$ \\
\hline Intelligence & $.21^{*}$ & .09 \\
1. Simultaneous Processing & $.41 \dagger$ & $.35 \dagger$ \\
2. Successive Processing & & \\
Achievement & $.58 \dagger$ & $.49 \dagger$ \\
3. Mathematics & $.51 \dagger$ & $.50 \dagger$ \\
4. Reading Recognition & $.54 \dagger$ & $.54{ }^{\dagger}$ \\
5. Reading Comprehension & $.44 \dagger$ & $.40 \dagger$ \\
6. Spelling & $.33 \dagger$ & $.41 \dagger$ \\
7. General Information & & \\
Language & $.43 \dagger$ & $.55 \dagger$ \\
8. PPVT & & \\
Note-PPVT, Peabody Picture Vocabulary Test. & ${ }^{*} p<.05$. & $\dagger p<.01$.
\end{tabular}

battery and the sentence span task were significantly related to individual differences in intelligence and achievement. Thus, the correlational pattern of the WM composite score was comparable to that in previous studies (e.g., Daneman \& Carpenter, 1980; Turner \& Engle, 1989); further, the composite score had sufficient criterion-related validity for testing the relationship between $W M$ and age-related differences in the next experiment.

\section{Discussion}

The results from Experiment 1 yielded four important findings. First, the WM measures are significantly correlated with a number of aptitude and achievement measures. Second, the WM tasks have reasonable intercorrelations. Although the intercorrelations are higher than those found in previous studies with adults (see Salthouse, 1990, for review), some tasks were weakly correlated (e.g., Tasks 1 and 11), suggesting that more than one factor may account for the correlations. Third, the WM battery has significant and substantial correlations with the sentence span task, supporting the construct validity of the WM measures. Finally, intercorrelations among working-memory measures remained significant even when age was partialed out. The findings suggest that, although the mechanisms that influence individual differences in WM may be accentuated with increases in age, a common system accounts for the intercorrelation at both an individual and age-related level of analysis.

\section{EXPERIMENT 2}

Although the results of Experiment 1 support previous studies on the generality of WM across task and processing conditions (e.g., Engle et al., 1992), the results are equivocal about whether general processing efficiency or general capacity underlie individual differences and age-related performance. Experiment 2 extends the earlier findings in two ways. First, Experiment 2 was designed to determine, via a factor analysis, whether the WM measures in Experiment 1 reflect distinct operations.
It might be argued that the high intercorrelation patterns among WM measures in Experiment 1 occurred because the tasks draw from the same pool of long-term memory resources. If tasks representing distinct operations should yield statistically comparable correlations with achievement measures, then stronger support for a generalized system (such as an executive processor) playing a role in WM performance could be inferred.

Second, Experiment 2 was designed to determine whether age-related and individual differences in WM are primarily functional or related to storage capacity. The contribution of different factors to children's working memory was investigated by using cues to bring the subject's performance to an asymptotic level. This approach implicitly assumes that if younger children are provided help in accessing previously presented information, then residual differences between younger and older children are due to the availability of the contents within a limited-capacity working-memory system. This assumption is bolstered by recent work on individual differences and working memory. For example, Cantor and Engle (1993) stated that "the content of WM is information in LTM [long-term memory] that has been activated above some critical threshold.... As the activation level of a concept increases, so does its accessibility (p. 1101). Cuing procedures increase activation to stored information, but this activation is limited by WM capacity. That is, individual differences are not driven by processing efficiency (Engle et al., 1992). Rather, as stated by Cantor and Engle (1993), "People differ in the total amount of activation available to retrieve information in LTM. This difference will be manifested in any task that makes at least moderate demands for such activation. High- and low-capacity subjects, as indexed by the WM spans, actually differ in their activation limits" (p. 1102). Thus, adapting systematic cuing procedures to the measurement of working memory does enhance our understanding of why development and individual differences emerge. For example, if younger children's working-memory deficits are due primarily to processing efficiency rather than storage capacity, then procedures that facilitate access to previously presented material ought to bring younger and older children to approximately the same asymptotic level. On the other hand, if younger children's workingmemory deficits are due to storage capacity, then procedures that facilitate access to previously presented information would not bring them to the same level of performance as older children. We conducted the second experiment to examine the validity of these assumptions.

To assess the relative contribution of processing or storage, children were presented WM tasks under initial, gain, and maintenance conditions. Initial conditions (noncued) were the same as those in Experiment 1, whereas the gain condition in Experiment 2 provided cues to maximize WM performance to its highest level. Maintenance conditions (i.e., maintaining the gain condition) assessed WM performance without cues (probes or hints). Based on these conditions, both a general-processing and a capacity model would be supported if the following events 
occurred: (1) Correlations between WM measures and achievement are not isolated to particular conditions or type of processing and (2) performance improves under cued conditions. The general-processing hypothesis would be supported, however, if measures of processing efficiency, such as the number of cues required to achieve improvement in WM performance, accounted for most of the variance in age-related performance. Further, if age-related differences in WM are due to less efficient processing, then younger children's WM performance should approximate older children's performance with cues. Thus, it would be expected that the number of cues (probes) used to establish gain performance would be an important predictor of age-related performance. This is because probes augment processing efficiency by facilitating access to previously presented information. In contrast, if age-related differences in WM are primarily due to a general constraint in storage capacity, then performance differences between age groups on gain and maintenance conditions would be accented compared to the initial testing conditions. Further, it would be expected that conditions that place high demands on storage (maintenance vs. initial conditions) would be a major predictor of age-related performance.

To further disentangle competing hypotheses related to whether age-related differences reflect a general or specific system, a model outlined by Hale (1990) and Kail (1993) was used. This model provides a means to separate out global processing from isolated processes. According to this model, the relative contribution of a global mechanism (e.g., executive storage system) is revealed by the linear relationship of one age group to the other across all processing conditions. A nonlinear function implies that residual differences between age groups are attributable to isolated processes (e.g., verbal or visualspatial resource systems), whereas a linear function implies that an age-related difference in performance is related primarily to the intrinsic efficiency of all individual processing components. In accordance with this model, it was predicted that if younger children's WM performance reflects a global storage system, their performance can be accurately predicted from the performance of older children.

\footnotetext{
Method

Subjects. The subjects were 156 children selected from various sites in rural and urban areas in British Columbia, California, Colorado, and Wyoming. These children were part of a standardization study (Swanson, 1995). All subjects were English speaking, considered normal achievers, and were not suffering from any handicapping condition. Sixty percent of the sample were female. Eighty-seven percent of the sample were Anglo, $8 \%$ were Hispanic, and $5 \%$ were Asian. The sample was divided into three groups on the basis of chronological age (CA): younger $(N=83 ; M \mathrm{CA}=$ $7.47, S D=1.18)$, middle $(N=36 ; M C A=10.25, S D=.56)$, and older $(N=37 ; M C A=13.44, S D=1.37)$.

Dependent measures. The same tasks as those in Experiment 1 were administered. Four dependent measures were calculated in this study in order to determine which measure best predicted age-related performance in WM. First, an initial score was determined on the basis of the number of items recalled without assis-
}

tance. Once an item was missed, a series of four cues or probes was presented to aid the child in recalling information. The cuing process continued until the most explicit hint (Cue 4) failed to help the child provide the correct response. Thus, the second measure, probe score, was the number of hints needed to achieve the gain score. A third measure, gain score, or asymptotic level, was defined as the highest score that was obtainable under probing conditions. A final measure, maintenance score, was defined as the stability of the asymptotic level after the probing conditions were removed. Once Tasks $1-6$ were administered, maintenance scores were determined. Maintenance scores for Tasks 7-11 were determined after the administration of Task 11. Maintenance scores were determined by presenting to the child the test item related to the gain score (items that produced the highest level of performance), but without the cues. If the child was able to maintain the same level of performance, the maintenance score was the same as his/her gain score. If the child failed the item, he/she was assigned the same score as the initial score.

Materials and Procedure. Each subject was tested individuaily. All examiners were trained in one 3-h session prior to the testing of children. Total testing time was approximately 90 min per student. All children were administered tasks in which initial, probe, gain, and maintenance scores were determined. All items on WM tasks were administered until probing did not improve performance. For some children who were not able to respond correctly to the process question (less than $2 \%$ of the sample), item recall was not requested and their response was scored as 0 .

A complete discussion and an example of probing procedures are provided in Swanson (1993). To summarize, the "bow shaped curve," commonly found in episodic memory studies, provides the basis for ordering a series of cues from implicit to explicit information. Cues are administered on the basis of the type of error made (i.e., whether the error was related to recency, primacy, or middle items), and cuing procedures continue until all targeted items cannot be recalled. The order of cues was based on the assumption that the first cue provides information about the final items because these items are the least susceptible to interference. The second cue was assumed to provide information about the primacy (first) items because they are the most reliant on long-term memory processes. The third cue provides additional information about the middlepresented items because these items are the most susceptible to interference and storage limitations. Finally, if the child fails to benefit from any of the previous three cues, all the items are repeated and retested. Probing procedures continue until all targeted items cannot be accessed (recalled). Because children were probed only about items for which they answered the process question correctly, it was assumed that poor item retrieval is attributable to item accessibility rather than to items not being adequately stored (Tulving \& Pearlstone, 1966)

Criterion measure. The Reading and Mathematics subtests from the Wide Range Achievement Test (Jastak \& Wilkinson, 1984) were used as the criterion measure. Means and standard deviations, by age group, are shown in Table 8 . The Reading subtest contains tasks of naming single words and the Mathematics subtest involves solving written computations. Median reliability for each subtest is .92. Wide Range Achievement Test scores are provided at the bottom of Table 8 .

\section{Results}

Age-related performance. The mean initial scores, gain scores, maintenance scores, and number of cues (probe score) required for performance as a function of age group are shown in Table 5. To determine if cuing procedures influenced performance at each age group, effect sizes were calculated. Effect sizes were calculated for gain scores as ES = (Mean of gain - mean of initial/ standard deviation of initial scores) and for maintenance 
scores as ES $=($ Mean of maintenance - mean of initial performance/standard deviation of initial scores). According to Cohen (1977), effect sizes less than or equal to .10 are small, those between .30 and .50 are medium, and those greater than .80 are large. As can be calculated from Table 5, cuing procedures had an impact on all tasks for all age groups. Mean effect sizes for the gain scores for 7-, 10-, and 13-year-olds were .94 ( $S D=.41)$, $1.21(S D=.58)$, and $1.30(S D=.50)$, respectively. Mean effect sizes for maintenance scores of 7-, 10-, and 13-year-olds were $.38(S D=.33), .69(S D=.40)$, and $.82(S D=.23)$, respectively. Overall, all age groups improved approximately 1 standard deviation in performance on the gain condition and approximately .5 standard deviation on the maintenance condition. The effect sizes also increased as children became older. For the subsequent analyses, all raw scores were converted to $z$-scores to control for the unequal range and subject variance between the tasks. [Because performance on some tasks may have proved difficult for younger subjects (therefore reducing correlations), a correction procedure for difficulty outlined by Daneman and Carpenter, 1980 , p. 458 , was used. However, the results of the analysis were comparable to the $z$-score transformation and therefore are not reported.]

As shown in Table 5, older children outperformed younger children on all WM measures. A multivariate analysis of variance (MANOVA) across WM tasks, as a function of age group, was significant for initial $[F(22,286)=$ $4.21, p<.001]$, gain $[F(22,286)=4.71, p<.001]$, probe $[F(22,286)=2.19, p<.001]$, and maintenance scores $[F(22,286)=5.62, p<.001]$. All univariates were significant $(p s<.01)$. A Duncan Multiple Range Test indicated that the general pattern of significant differences was that the older age group had higher WM scores than the younger ages, and the youngest age group had the lowest performance overall. To determine the major source of age differences, composite scores $(z$-scores summed across WM tasks) were computed for initial, gain, probe, and maintenance conditions. These scores were entered into a forward stepwise regression analysis in which the criterion variable was age. The results indi-

Table 5

Initial, Gain, Probe, and Maintenance Scores as a Function of Group

\begin{tabular}{|c|c|c|c|c|c|c|c|c|}
\hline \multirow[b]{2}{*}{ Subtest } & \multicolumn{2}{|c|}{ Initial } & \multicolumn{2}{|c|}{ Gain } & \multicolumn{2}{|c|}{ Probes } & \multicolumn{2}{|c|}{ Maintenance } \\
\hline & $M$ & $S D$ & $M$ & $S D$ & $M$ & $S D$ & $M$ & $S D$ \\
\hline \multicolumn{9}{|c|}{ Raw Scores for 7-Year-Olds } \\
\hline 1. Rhyming & 1.73 & 1.15 & 2.80 & 1.16 & 3.08 & 2.56 & 2.40 & 1.25 \\
\hline 2. Visual Matrix & 2.73 & 1.40 & 4.30 & 1.84 & 3.73 & 4.17 & 3.20 & 1.60 \\
\hline 3. Auditory Digit Sequence & 1.64 & 1.19 & 3.45 & 1.41 & 4.98 & 4.21 & 2.45 & 1.48 \\
\hline 4. Mapping & 1.63 & 1.15 & 2.84 & 1.34 & 2.73 & 2.79 & 2.24 & 1.18 \\
\hline 5. Story Recall & 2.45 & 2.57 & 4.00 & 3.42 & 1.49 & 2.71 & 3.08 & 2.98 \\
\hline 6. Picture Sequence & 1.34 & 1.17 & 2.09 & 1.28 & 1.98 & 2.54 & 1.62 & 1.20 \\
\hline 7. Phrase & .87 & .93 & 2.60 & 1.30 & 4.36 & 2.92 & 1.86 & 1.39 \\
\hline 8. Spatial Organization & 1.20 & 1.04 & 1.90 & 1.55 & 1.27 & 4.27 & 1.1 & 1.35 \\
\hline 9. Semantic Association & 1.16 & 1.34 & 2.67 & 2.04 & 4.23 & 4.39 & 1.17 & 1.81 \\
\hline 10. Semantic Categorization & 1.52 & 1.33 & 2.59 & 1.24 & 2.99 & 2.74 & 2.19 & 1.37 \\
\hline 11. Nonverbal Sequencing & 1.70 & 2.79 & 3.09 & 4.66 & .60 & 1.98 & 2.09 & 3.59 \\
\hline \multicolumn{9}{|c|}{ Raw Scores for 10-Year-Olds } \\
\hline 1. Rhyming & 2.06 & 1.24 & 3.80 & 1.50 & 4.26 & 4.45 & 2.75 & 1.44 \\
\hline 2. Visual Matrix & 3.36 & 1.49 & 5.16 & 1.68 & 4.15 & 3.73 & 4.19 & 1.72 \\
\hline 3. Auditory Digit Sequence & 2.15 & 1.29 & 4.52 & 1.74 & 5.98 & 3.64 & 3.58 & 1.81 \\
\hline 4. Mapping & 1.54 & 1.35 & 2.00 & 1.43 & 3.52 & 1.73 & 3.24 & 3.25 \\
\hline 5. Story Recall & 4.06 & 2.49 & 6.19 & 2.64 & .97 & 1.62 & 5.00 & 2.48 \\
\hline 6. Picture Sequence & 1.64 & 1.21 & 2.75 & 1.46 & 1.67 & 1.90 & 2.16 & 1.29 \\
\hline 7. Phrase & 1.18 & .94 & 3.25 & 1.25 & 5.29 & 3.16 & 2.11 & 1.32 \\
\hline 8. Spatial Organization & 1.87 & 1.26 & 3.02 & 1.94 & 2.20 & 5.21 & 2.16 & 1.15 \\
\hline 9. Semantic Association & 1.59 & 1.11 & 3.80 & 1.72 & 4.95 & 3.26 & 3.02 & 1.94 \\
\hline 10. Semantic Categorization & 1.81 & 1.26 & 3.22 & 1.69 & 3.62 & 2.76 & 2.64 & 1.62 \\
\hline 11. Nonverbal Sequencing & 4.13 & 6.18 & 8.00 & 9.86 & 5.45 & 1.35 & 5.02 & 7.54 \\
\hline \multicolumn{9}{|c|}{ Raw Scores for 13-Year-Olds } \\
\hline 1. Rhyming & 2.39 & 1.12 & 3.89 & 1.39 & 3.68 & 3.00 & 3.27 & 1.22 \\
\hline 2. Visual Matrix & 3.98 & 1.37 & 6.27 & 2.16 & 4.44 & 3.85 & 5.29 & 2.02 \\
\hline 3. Auditory Digit Sequence & 3.12 & 1.23 & 5.35 & 1.18 & 5.54 & 4.16 & 4.48 & 1.70 \\
\hline 4. Mapping & 2.62 & 1.52 & 4.81 & 1.57 & 4.66 & 3.26 & 4.05 & 1.82 \\
\hline 5. Story Recall & 6.39 & 2.62 & 8.10 & 2.73 & 1.24 & 2.41 & 7.35 & 2.92 \\
\hline 6. Picture Sequence & 2.33 & 1.24 & 3.78 & 1.88 & 3.24 & 3.60 & 2.83 & 1.34 \\
\hline 7. Phrase & 1.79 & 1.41 & 4.29 & 1.56 & 6.25 & 3.93 & 3.83 & 1.70 \\
\hline 8. Spatial Organization & 2.43 & 1.87 & 3.81 & 1.76 & 2.73 & 5.64 & 3.12 & 1.84 \\
\hline 9. Semantic Association & 2.14 & 1.36 & 4.59 & 1.36 & 5.52 & 2.77 & 3.97 & 1.65 \\
\hline 10. Semantic Categorization & 2.31 & 1.05 & 4.00 & 1.22 & 4.47 & 2.92 & 3.54 & 1.38 \\
\hline 11. Nonverbal Sequencing & 5.87 & 6.38 & 8.27 & 8.01 & 1.42 & 2.73 & 7.37 & 7.82 \\
\hline
\end{tabular}


Table 6

Fit Statistics for Each Age Group

\begin{tabular}{cccc}
\hline & \multicolumn{3}{c}{ Parameters } \\
\cline { 2 - 4 } Age Group & Slope & Intercept & $R^{2}$ \\
\hline & Criterion Group $=13$-Year-Olds \\
7-year-olds & 1.53 & .86 & $.57^{*}$ \\
10 -year-olds & 1.09 & .73 & $.86^{*}$ \\
& Criterion Group & $=10$-Year-Olds & \\
7-year-olds & 1.33 & .26 & $.56^{*}$ \\
${ }^{*} p<.01$. & & &
\end{tabular}

cated that the single best contributor to age is the maintenance of optimal performance $\left[R^{2}=.48, F(1,151)=\right.$ $138.78, p<.0001]$. No other variable entered significantly $(p s>.05)$ into the equation. Thus, scores related to the maintenance of WM, after optimal training conditions, accounted for approximately $50 \%$ of the variance.

Analysis across tasks and conditions. Regardless of processing conditions, the previous analyses indicated that older children outperformed younger children on working-memory tasks. Further, younger children's inferior performance relative to that of older children cannot be compensated for by the administration of systematic probes (cues). The results also indicated that high-load conditions (maintenance conditions) were the best predictor of age-related performance. Overall, the findings suggest that younger children's depressed performance reflects a general WM system rather than problems related to isolated processes. Further support for this interpretation would be found, however, if a clear linear relationship could be shown to exist between age groups across all memory conditions. To test this possibility, a mathematical technique outlined by Hale (1990) and Kail (1993) was used to compare the younger age group with the older age group. According to the hypothesis that a global mechanism is responsible for age-related changes in WM, the correlation across WM conditions between younger and older subjects should approximate 1.0. That is, the closer a child gets to maturity, the higher the $R^{2}$ between the criterion group (older subjects) and the age closest to the criterion group. Furthermore, the slope of the function of younger children to older children from the corresponding experimental conditions provides an estimate of WM span. That is, the slope changes exponentially with age (see Kail, 1993, for discussion).

For each of the treatment conditions (initial, gain, maintenance; verbal vs. visual-spatial), the mean performances of each given younger age group were plotted as a function of the older age group in the corresponding WM condition. Table 6 shows the fit between comparison groups. As shown, the relations between the younger and older groups are linear. Notice also that these fits are extremely good for the 10- and 13-year-olds, as indicated by the high $r^{2}$ values. When compared to the fit between 7-year-olds and 13-year-olds, the better fit between 10 - and 13-year-olds indicates a gradual approximation to older children's processing. In general, the results support the notion that all task conditions contributing to WM performance are affected by age group, and that age-related performance is not differentially affected by isolated processing (verbal, visual-spatial; initial vs. gain vs. maintenance) conditions. Thus, the earlier reported differences between the age groups appear to be related to a general WM system.

Individual differences. This analysis was conducted to determine whether individual differences in WM within age groups reflect a general or specific system. Before one can test whether individualized differences in WM reflect a generalized or specific system, however, it is necessary to establish that the WM measures reflect independent operations. This was done in two ways. First, it was necessary to determine if the magnitude of the intercorrelations between WM measures was merely a function of age. The average intercorrelation between initial scores with $(r=.38)$ and without $(r=.46)$ age partialed out was not significant $(Z=.90, p>.05)$, suggesting that the intercorrelation patterns are not merely a function of age. Second, unique components related to initial scores were analyzed via a principal components factor analysis with the effects of age partialed out. Those component scores were then correlated with achievement. The principal factor analysis, with a varimax rotation, is shown in Table 7. Two criteria were used to determine the number of factors: the Kaiser-Guttman rule of extracting only factors with eigenvalues greater than 1 and Cattell's scree test. According to these two criteria for factor acceptance, both criteria support a two-factor model. The varimax rotations redistributed the total common variance of 5.74 into roughly equal portions for two factors. The two factors parallel the possible contribution of semantic and episodic resources in WM processing (see Swanson, 1992, for discussion). However, this interpretation is not obligatory for our findings. The results do support, however, the notion that the WM measures tap at least two distinct operations.

Table 8 shows the Pearson product moment correlation coefficients between the two factor scores and achievement as a function of age group. Consistent with previous studies (Daneman \& Tardiff, 1987; Engle et al., 1992), correlations for composite scores related to visual-spatial and verbal WM tasks were also included in the analysis. One can draw two general conclusions from the results shown in Table 8. First, as expected, there is an age-related trend in the relationship between WM and achievement. Higher coefficients emerge for 10- and 13-year-olds compared to 7-year-olds. Second, there is weak support for the notion that specific processes are more directly related to one academic domain than another. More specifically, a comparison of verbal and visual-spatial WM correlations related to math and reading did not reveal any significant differences (Fisher $z$-score comparison) in the magnitude of the coefficients $(p>.05)$ across the age groups. One exception was found for the 13-yearolds, for whom the magnitude of the coefficient for the 
Table 7

Intercorrelations and Factor Analyses (Varimax Rotation) for Initial Scores

\begin{tabular}{|c|c|c|c|c|c|c|c|c|c|c|c|c|c|c|}
\hline \multirow[b]{2}{*}{ Measures } & \multicolumn{2}{|c|}{ Factor } & \multirow[b]{2}{*}{1} & \multirow[b]{2}{*}{2} & \multirow[b]{2}{*}{3} & \multirow[b]{2}{*}{4} & \multirow[b]{2}{*}{5} & \multirow[b]{2}{*}{6} & \multirow[b]{2}{*}{7} & \multirow[b]{2}{*}{8} & \multirow[b]{2}{*}{9} & \multirow[b]{2}{*}{10} & \multirow[b]{2}{*}{11} & \multirow[b]{2}{*}{12} \\
\hline & 1 & II & & & & & & & & & & & & \\
\hline 1. Rhyming & .78 & .06 & & .32 & .51 & .42 & .26 & .36 & .52 & .29 & .48 & .45 & .10 & .23 \\
\hline 2. Visual Matrix & .44 & .59 & & & .53 & .54 & .48 & .47 & .38 & .30 & .46 & .40 & .28 & .23 \\
\hline 3. Auditory Digit Sequence & .67 & .38 & & & & .56 & .54 & .53 & .52 & .38 & .54 & .54 & .23 & .38 \\
\hline 4. Mapping & .57 & .40 & & & & & .43 & .48 & .53 & .36 & .42 & .40 & .20 & .25 \\
\hline 5. Story Recall & .27 & 61 & & & & & & .47 & .36 & .40 & .39 & .38 & .27 & .38 \\
\hline 6. Picture Sequence & .48 & .54 & & & & & & & .45 & .34 & .50 & .48 & .30 & .24 \\
\hline 7. Phrase Sequence & .78 & .12 & & & & & & & & .41 & .56 & .52 & .15 & .24 \\
\hline 8. Spatial/Organization & .41 & .35 & & & & & & & & & .36 & .38 & .21 & .23 \\
\hline 9. Semantic Association & .68 & .34 & & & & & & & & & & .63 & .29 & .25 \\
\hline 10. Semantic Categorization & .69 & .26 & & & & & & & & & & & .24 & .21 \\
\hline 11. Nonverbal Sequencing & .02 & .74 & & & & & & & & & & & & .24 \\
\hline
\end{tabular}

visual component was twice as large as the verbal component coefficient for math performance. However, coefficients were comparable between visual-spatial and verbal components for this age group for the domain of reading.

\section{Discussion}

Experiment 2 focused on whether a general system or a task-specific system underlies age-related and individual differences in WM performance and yielded two important findings. First, two independent operations captured the memory tasks, but those operations (factors) yielded comparable correlation patterns to reading and mathematics across and within age groups. Second, agerelated differences were better predicted from highdemand (maintenance) than low-demand (initial) conditions or general-processing measures (probe scores). Further, the maintenance conditions contributed approximately $50 \%$ of the variance in age-related performance, suggesting that a general-capacity system is in operation. Younger children's performance was a linear func-

Table 8

Correlations Between Working-Memory Measures and Achievement for 7-, 10-, and 13-Year-Olds

\begin{tabular}{|c|c|c|c|c|c|c|}
\hline & \multicolumn{3}{|c|}{ Math } & \multicolumn{3}{|c|}{ Reading } \\
\hline & 7 & 10 & 13 & 7 & 10 & 13 \\
\hline \multicolumn{7}{|l|}{ Initial Measures } \\
\hline Total score & $.24^{*}$ & $.55 \dagger$ & $.65 \dagger$ & $.34 \dagger$ & $.56 \dagger$ & $.44 \dagger$ \\
\hline Composite-semantic & .15 & $.51 \dagger$ & $.50 \dagger$ & $.23^{*}$ & $.59 \dagger$ & $.37^{*}$ \\
\hline Composite-episodic & $.24^{*}$ & $.40 \dagger$ & $.52 \dagger$ & $.33 t$ & $.35^{*}$ & .32 \\
\hline \multicolumn{7}{|l|}{ Component Scores } \\
\hline Visual-spatial & $.23 *$ & $.46 \dagger^{\dagger}$ & $.72 \dagger$ & $.25^{*}$ & $.42 \dagger$ & $.34^{*}$ \\
\hline \multirow[t]{3}{*}{ Verbal } & .20 & $.45 \dagger$ & .32 & $.34 \dagger$ & $.51 \dagger$ & $.41 \dagger$ \\
\hline & \multicolumn{6}{|c|}{ WRAT-R Mean Raw Scores } \\
\hline & $M$ & $S D$ & & $M$ & $S D$ & \\
\hline Age I & 15.66 & 8.30 & & 34.51 & 19.90 & \\
\hline Age II & 24.00 & 13.91 & & 56.82 & 18.48 & \\
\hline Age III & 20.31 & 10.40 & & 50.11 & 14.83 & \\
\hline
\end{tabular}

Note-WRAT-R, Wide Range Achievement Test-Revised. Scores for Age III are from Level II; Age II are prorated scores from Levels I and II; and Age I scores are from Level I of the WRAT-R. ${ }^{*} p<.05$. $\dagger p<.01$. tion of older children's, suggesting that age-related differences are a function of global processes. Overall, the results suggest that the sources of individual and agerelated differences are related to a general rather than a specific processing system.

\section{GENERAL DISCUSSION}

The present study produced two important findings. First, the relationship between WM and aptitude/achievement measures in children's performance is not task specific. As predicted by the general-processing model, diverse WM tasks predicted individual and age-related differences in children's achievement independently of the type of processing task in which they were embedded. Similar results have been demonstrated with adult samples (e.g., Cantor et al., 1991; Salthouse et al., 1989; Turner \& Engle, 1989).

Second, the results suggest that it would be premature to reject a general-capacity model as an explanation of age-related and individual differences in children's WM for at least two reasons. First, enhanced processing produced a greater age effect than initial scores, which is the reverse of the finding predicted by a processing limitation hypothesis (in that cues were intended to alleviate such problems). Second, the number of probes necessary to establish asymptotic performance, an assumed measure of processing efficiency, was not an important predictor of age-related differences. Rather, maintenance of WM performance, after the removal of probes, best contributed to age-related performance. Experiment 2 indicated that high-demand WM conditions accounted for almost $50 \%$ of the variance in age-related performance. It was assumed that maintenance conditions required more storage capacity than initial conditions, especially because asymptotic performance was not bolstered by probes. The finding that a child's ability to sustain asymptotic performance (maintenance condition), rather than processing efficiency (number of cues necessary to establish asymptotic performance), best predicts age-related performance suggests that a general-capacity system may be responsible for variations in WM performance. 
The studies also addressed the question of whether the general processes that influence age-related differences and individual differences are comparable. A comparison of the results reflective of age-related performance with performance related to individual differences reveals that (1) age effects on working memory performance are best captured after brief training occurs (Experiment 2 ), (2) age effects were not isolated to verbal or visualspatial working-memory measures (Experiments 1 and 2 ), and (3) the correlations between working memory and measures of reading follow the same correlational pattern as that found between measures of mathematics and working memory across age (Experiment 2 ). The important findings related to individual differences were that (1) the mean intercorrelations among working-memory measures remained significant with age partialed out (Experiments 1 and 2) and (2) the magnitude of the correlation between working memory and achievement was statistically comparable across age groups (Experiment 2). The simplest interpretation of age-related and individual difference is that to some degree the same mechanisms affect both findings. Although older children have better storage or concurrent processing and thus more general resources available for processing information than younger children, comparable correlational patterns between diverse WM measures and achievement occur within age groups.

Two major alternative interpretations of the results must be considered. The first option is related to the increasing skepticism about the usefulness of resourceallocation models that invoke a central processing mechanism (see, e.g., Brainerd \& Reyna, 1989). This option suggests that younger children are less resistant to interference (see Brainerd \& Reyna, 1993, and Dempster, $1993 \mathrm{~b}$, for discussion of this model). For example, it might be argued that the process questions in the current tasks constituted a very temporary competing condition with storage. Further, the working-memory tasks varied considerably in terms of the number of processing questions (e.g., compare the story task with the digit-recall task) the subjects were exposed to, and the pervasiveness of younger children's poor performance across such diverse measures may reflect a general interference condition. As a consequence, younger children might have had difficulty preventing unnecessary information from entering working memory and, therefore, were more likely to consider alternative interpretations of material (such as asked for in the processing questions) that were not central to the task. This interpretation fits within several recent models that explain individual differences in memory performance in terms of inhibitory mechanisms (e.g., Brainerd \& Reyna, 1989, 1993; Dempster, 1993a, 1993b; Hasher \& Zacks, 1988), without positing some form of a capacity deficit.

Although I see the above model as a viable alternative to the results, I have three reservations. First, only subjects who answered the process question correctly were analyzed. That is, if a process question was missed, the child was not asked to recall previously stored information. This procedure is different from those of previous studies (e.g., Daneman \& Carpenter, 1980), which have allowed a dissociation between the process question (i.e., it is not necessary for subjects to answer the process question correctly) and the retrieval question. This control provides feedback to subjects related to the interpretation and/or relevance of the material to be remembered. Thus, there was an experimenter-imposed association between the process and retrieval question for the same set of inputs. Further, a post hoc analysis of the protocols did not indicate that irrelevant information from the process question was more likely recalled by younger than older children. That is, if individual differences in children's performance are due to an ability to resist interference for irrelevant items, then there would be substantial evidence of irrelevant information recall between age groups. Such was not the case in this study. Second, if younger children suffer more interference (i.e., diminished inhibition in that a large number of traces are simultaneously active) than older children, then one would expect the probing to narrow significantly the alternative interpretations of items in memory compared to older children. That is, inefficiencies in inhibiting traces or competition effects should be reduced more in younger than older children. Further, one would predict that a procedure that gives feedback on the relevancy of a response should lead to a substantial increase in memory performance in the group with the diminished inhibitory efficiency. An analysis of effect sizes related to the gain condition, however, indicates statistically comparable changes between age groups. Finally, it seems to me that the concept of "interference" can be tied to a resourceallocation model. That is, capacity constraints may underlie individual differences in inhibitory efficiency. This has been suggested in the literature on depression and working memory (e.g., Ellis, 1990), as well as on aging and working memory (e.g., Hasher, Stoltzfus, Zacks, \& Rypma, 1991). In short, younger children may use more capacity than older children to inhibit or resist potential interference for irrelevant items.

The second option for interpreting the results suggests that younger children suffer from functional workingmemory problems (e.g., a lack of flexibility in coordinating various memory stores) rather than processing constraints related to a particular store. In this view, the executive processing system of younger subjects may or may not have the same storage capacity, but an important source of individual and developmental differences in children is an inability to coordinate and/or compensate for the processes they have. This option differs from the processing versus storage issue by emphasizing the coordination of processes. It also differs from the inhibition of irrelevant information view, discussed above. In support of this option, Yee, Hunt, and Pellegrino (1991) have argued that complex tasks (i.e., dual- or multipletask situations) are more likely to reflect the coordination of processes than divided attention between compet- 
ing memory traces (see also Carlson, Wenger, \& Sullivan, 1993, for a testing of this notion). Thus, although individual differences in working memory may reflect a situation in which information is poorly encoded and maintained, individual differences also occur in how subjects switch between and coordinate sources of information. For example, younger children may be forced to represent word information in a visual-spatial form, rather than leave it in an articulatory form, or vice versa, and this coordination across different representations is particularly difficult for younger children. I believe, however, that the major limitation of this interpretation for the present results is that it does not eliminate a resource-allocation model. This is because one can speculate that resource trade-offs can exist between storage and response execution processes (Carlson et al., 1993). Simply stated, there is a cost in switching and/or coordinating across multiple memory traces.

In summary, two experiments found that diverse WM tasks are reasonably intercorrelated, suggesting that they possess some commonality. It was also found that performance under conditions that place high demands on working memory are more predictive of age differences than conditions that enhance processing efficiency. The findings raise questions about whether a processingefficiency hypothesis provides an adequate explanation of individual and age-related differences in children's working-memory performance. No doubt, future research must focus on the interaction between executive and lower order processing during the act of reading to disentangle the possible alternative interpretations of the results. The results clearly suggest, however, that younger children experience a generalized deficit in working memory related to the preservation of new information. These deficits emerged across conditions that place demands on working-memory performance, suggesting that the source of individual differences may be related to generalized processing constraints.

\section{REFERENCES}

BADDELEY, A. D. (1986). Working-memory. London: Oxford University Press.

BJoRKLund, D. F., \& HaRnishfeger, K. K. (1990). The resources construct in cognitive development: Diverse sources of evidence and a theory of inefficient inhibition. Developmental Review, 10, 48-71.

BRAINERD, C. J., \& REYNA, V. F. (1989). Output-interference theory of dual-task deficits in memory development. Journal of Experimental Child Psychology, 47, 1-18.

Brainerd, C. J., \& Reyna, V. F. (1990). Gist is the grist: Fuzzy-trace theory and perceptual salience effect in cognitive development. Developmental Review, 10, 3-47.

Brainerd, C. J., \& Reyna, V. F. (1993). Domains of fuzzy trace theory. In M. L. Howe \& R. Pasnak (Eds.), Emerging themes in cognitive development: Foundations (Vol.1, pp. 50-93). New York: Springer-Verlag.

Cantor, J., \& EnGle, R. W. (1993). Working memory capacity as long-term memory activation: An individual-differences approach. Journal of Experimental Psychology: Learning, Memory, \& Cognition, 19, 1101-1114.

Cantor, J., Engle, R. W., \& Hamilton, G. (1991). Short-term memory, working-memory, and verbal abilities: How do they relate? Intelligence, 15, 229-246.
Carlson, R. A., Wenger, J. L., \& Sullivan, M. A. (1993). Coordination information from perception and working memory. Journal of Experimental Psychology: Human Perception \& Performance, 19, 531-548.

CASE, R. (1985). Intellectual development: Birth to adulthood. New York: Academic Press.

Case, R., Kurland, D. M., \& Goldberg, J. (1982). Operational efficiency and the growth of short-term memory span. Journal of Experimental Child Psychology, 33, 386-404.

Chapman, M., \& Lindenberger, U. (1992). How to detect reasoning remembering dependence (and how not to). Developmental Review, 12, $187-198$

CoHen, J. (1977). Statistical power analysis for the behavioral sciences. New York: Academic Press.

DANEMAN, M. (1987). Reading and working-memory. In J. R. Beech \& A. M. Colley (Eds.), Cognitive approaches to reading (pp. 57-86). New York: Wiley.

Daneman, M., \& Carpenter, P. A. (1980). Individual differences in working memory and reading. Journal of Verbal Learning \& Verbal Behavior, 19, 450-466.

Daneman, M., \& Green, I. (1986). Individual differences in comprehending and producing words in context. Journal of Memory \& Language, 25, 1-18.

Daneman, M., \& TaRdifF, T. (1987). Working memory and reading skill reexamined. In M. Coltheart (Ed.), Attention and performance XII: The psychology of reading (pp. 491-508). East Sussex, England: Erlbaum.

DemPSTER, F. N. (1985). Short-term memory development in childhood and adolescence. In C. J. Brainerd \& M. Pressley (Eds.), Basic processes in memory (pp. 209-248). New York: Springer-Verlag.

Dempster, F. N. (1993a). Resistance to interference: Developmental changes in a basic processing mechanism. In M. L. Howe \& R. Pasnak (Eds.), Emerging themes in cognitive development: Foundations (Vol. 2, pp. 3-27). New York: Springer-Verlag.

DEMPSTER, F. N. (1993b). Resistance to interference: Toward a unified theory of cognitive development and aging. Developmental Review, 12, 45-75.

DunN, L. M. (1981). Peabody picture vocabulary test. Circle Pines, MN: American Guidance Service.

Dunn, L. M., \& Markwardt, F. C. (1989). Peabody individual achievement test. Circle Pines, MN: American Guidance Service.

Ellis, H. C. (1990). Depressive deficits in memory: Processing initiative and resource allocation. Journal of Experimental Psychology: General, 119, 60-62.

Engle, R. W., Cantor, J., \& Carullo, J. J. (1992). Individual differences in working memory and comprehension: A test of four hypotheses. Journal of Experimental Psychology: Learning, Memory, \& Cognition, 18, 972-992.

HALE, S. (1990). A global developmental trend in cognitive processing speed in children. Child Development, 61, 653-663.

HaMmill, D. D. (1985). Detroit tests of learning aptitude-Second edition. Austin, TX: PRO-ED

Hasher, L., Stoltzfus, E. R., Zacks, R. T., \& Rypma, B. (1991). Age and inhibition. Journal of Experimental Psychology: Learning, Memory, \& Cognition, 17, 163-169.

HASHER, L., \& ZACKS, R. T. (1988). Working memory, comprehension and aging: A review and a new view. In G. H. Bower (Ed.), The psychology of learning and motivation (Vol. 22, pp. 193-225). San Diego: Academic Press.

Howe, M. L., \& Rabinowitz, M. (1990). Resource panacea? Or just another day in the developmental forest. Developmental Review, 10, 125-154.

Howe, M. L., \& Rabinowitz, M. (1991). Gist another panacea? Or just another day in the developmental forest. Developmental Review, 11, 305-316.

Howe, M. L., Rabinowitz, M., \& Grant, M. J. (1993). On measuring (in)dependence of cognitive processes. Psychological Review, 100, 737-747

JASTAK, J. J., \& WiLKInSON, G. (1984). Manual: The Wide Range Achievement Test-Revised. Wilmington, DE: Jastak Associates.

Just, M., \& Carpenter, P. A. (1992). A capacity theory of comprehension: Individual differences in working memory. Psychological Review, 99, 122-149. 
KafL, R. (1993). The role of global mechanisms in developmental change in speed of processing. In M. L. Howe \& R. Pasnak (Eds.), Emerging themes in cognitive development: Foundations (Vol. 1, pp. 97-116). New York: Springer-Verlag.

Kaufman, A., \& KaUfMaN, N. L. (1983). Kaufman assessment battery for children. Circle Pines, MN: American Guidance Service.

Morris, R., Craik, F., \& GICK, M. L. (1990). Age differences in working memory: The role of secondary memory and the central executive. Quarterly Journal of Experimental Psychology, 42A, 67-86.

SALTHOUSE, T. A. (1990). Working-memory as a processing resource in cognitive aging. Developmental Review, 10, 101-124.

SALTHOUSE, T. A., \& BABCOCK, R. (1991). Decomposing adult age differences in working memory. Developmental Psychology, 27, 763-776.

Salthouse, T. A., Mrtchell, D. R. D., Skovronek, E., \& Babcock, R. L. (1989). Effects of adult age and working-memory on reasoning and spatial abilities. Journal of Experimental Psychology: Human Learning \& Cognition, 15, 507-516.

Swanson, H. L. (1992). The generality and modifiability of working memory in skilled and less skilled readers. Journal of Educational Psychology, 84, 473-488.
SWANSON, H. L. (1993). Working memory in learning disability subgroups. Journal of Experimental Child Psychology, 56, 87-114. SWANSON, H. L. (1995). Cognitive processing test. Austin, TX: PRO-ED. Swanson, H. L., Cochran, K., \& EWERs, C. (1989). Working-memory and reading disabilities. Journal of Abnormal Child Psychology, 17, 745-756.

Tulving, E., \& Pearlstone, Z. (1966). Availability versus accessibility of information in memory for words. Journal of Verbal Learning \& Verbal Behavior, 5, 381-391.

TURNER, M. L., \& ENGLE, R. W. (1989). Is working-memory capacity task dependent? Journal of Memory \& Language, 28, 27-154.

Yee, P. L., Hunt, E., \& Pellegrino, J. W. (1991). Coordinating cognitive information: Task effects and individual difference in integrating information from several sources. Cognitive Psychology, 23, 615-680.

(Manuscript received January 18, 1995; accepted for publication February 10, 1995.) 